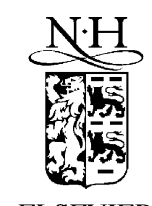

ELSEVIER

\title{
An efficient decoding technique for Huffman codes
}

\author{
Rezaul Alam Chowdhury ${ }^{a}{ }^{\text {* }}$, M. Kaykobad ${ }^{a}$, Irwin King ${ }^{b}$ \\ ${ }^{a}$ Department of Computer Science and Engineering, Bangladesh University of Engineering and Technology, Dhaka-1000, Bangladesh \\ ${ }^{\mathrm{b}}$ Department of Computer Science and Engineering, The Chinese University of Hong Kong, Hong Kong, People's Republic of China
}

Received 11 December 2000; received in revised form 21 May 2001

Communicated by F.B. Schneider

\begin{abstract}
We present a new data structure for Huffman coding in which in addition to sending symbols in order of their appearance in the Huffman tree one needs to send codes of all circular leaf nodes (nodes with two adjacent external nodes), the number of which is always bounded above by half the number of symbols. We decode the text by using the memory efficient data structure proposed by Chen et al. [Inform. Process. Lett. 69 (1999) 119-122]. (C 2002 Elsevier Science B.V. All rights reserved.
\end{abstract}

Keywords: Static Huffman code; Data structures; Complete binary tree

\section{Introduction}

Since the discovery of the Huffman encoding scheme [4] in 1952, Huffman codes have been widely used in the efficient storing of data, image and video [2]. Huffman coding has been subjected to numerous investigations in the past 50 years. Schack [7] considered the distribution of the length of a typical Huffman codeword, while Katona and Nemetz [5] studied the connection between the self-information of a source letter and its codeword length in a Huffman code. A traditional Huffman scheme collects frequency of occurrence of different symbols to be coded in the first pass, constructs a tree based upon which symbols receive codes. Then in the second pass symbols are coded and sent to the receiver. However, along with codes corresponding to symbols the Huffman tree is

\footnotetext{
${ }^{*}$ Corresponding author.

E-mail addresses: shaikat2@yahoo.com, shaikat@bdonline.com (R.A. Chowdhury), audwit@bdonline.com (M. Kaykobad), king@cse.cuhk.edu.hk (I. King).
}

also sent. There is a single pass version of Huffman coding called dynamic Huffman coding [8]. In the latter case symbols are coded according to the frequencies of symbols sent so far, and the tree is updated by both sender and receiver using the same algorithm. In this paper we consider efficient decoding of the socalled static Huffman code.

Hashemian [3] presented an algorithm to speed up the search process for a symbol in a Huffman tree and reduce the memory size. He proposed a treeclustering algorithm to avoid sparsity of Huffman trees. Finding the optimal solution of this clustering problem is still open. Later, Chung [2] gave a data structure requiring memory size of only $2 n-3$ to represent the Huffman tree, where $n$ is the number of symbols. Chen et al. [1] improved the data structure further to reduce the memory requirement to $\lceil 3 n / 2\rceil+$ $\lceil(n / 2) \log n\rceil+1$. In our scheme in addition to sending information on symbols we send information on all circular leaf nodes (nodes with two adjacent external nodes), that uniquely determines a Huffman tree. 
Our memory requirement is better than any existing data structure since the number of circular nodes is bounded above by $\lceil n / 2\rceil$. So in the worst case our memory requirement is $\lceil 3 n / 2\rceil$. In fact, the number of circular leaf nodes of a Huffman tree is 1 more than the number of nodes with 2 internal son nodes. Such a saving in the representation of Huffman trees is very important in the context of repeated Huffman coding [6], where the ultimate compression ratio depends upon the efficiency of representation of the Huffman tree. In our case decoding of codes is based upon Chen's data structure for storing the Huffman tree. While Chen et al. [1] addressed the problem of efficient decoding of a Huffman code, we address the problem of decoding streams of bits not knowing the length of bit patterns that correspond to a symbol. For clarity of presentation we first describe the idea of Chen et al. [1] and their data structure.

Let $T$ be a Huffman tree, which contains $n$ symbols. The symbols correspond to leaves of $T$ and are labeled from left to right as $s_{0}, s_{1}, \ldots, s_{n-1}$. The root is said to be at level 0 . The level of any other node is 1 more than the level of its father. The largest level is the height $h$ of the Huffman tree. The weight of a symbol is defined to be $2^{h-l}$, where $h$ is the height of the tree and $l$ is the level of a node corresponding to a symbol. Let $w_{i}$ be the weight of symbol $s_{i}, i=0, \ldots, n-1$. Let $f_{i}$ denote the cumulative weight up to weight $w_{i}$. Then $f_{0}=w_{0}, f_{i}=f_{i-1}+w_{i}$, for $i=1,2, \ldots, n-1$.

While Chen et al. [1] give an algorithm for decoding a codeword, and give the correct symbol as output or say that the given codeword does not correspond to any symbol, we address the problem of decoding the bit streams sent by the sender according to the Huffman tree that is available with the receiver in the data structure mentioned above. The above data structure requires an overhead of sending $n$ symbols and $n$ integers corresponding to them. This overhead can also be further reduced by the following.

If $T$ is a Huffman tree of height $h$, then $T^{\prime}$, the truncated Huffman tree, is obtained by removing all leaves (square nodes) and will have height $h-1 . T^{\prime}$ uniquely determines the Huffman tree $T$ since symbols correspond to left and right son nodes of the circular leaf nodes and the other sons of other single-son circular nodes. Then Huffman codes, representing circular leaf nodes of $T^{\prime}$ uniquely determines $T$. If there are $m$ circular leaf nodes, $m$ codewords corresponding to them, and $n$ symbols in order of appearance in the tree will suffice to represent the Huffman tree.

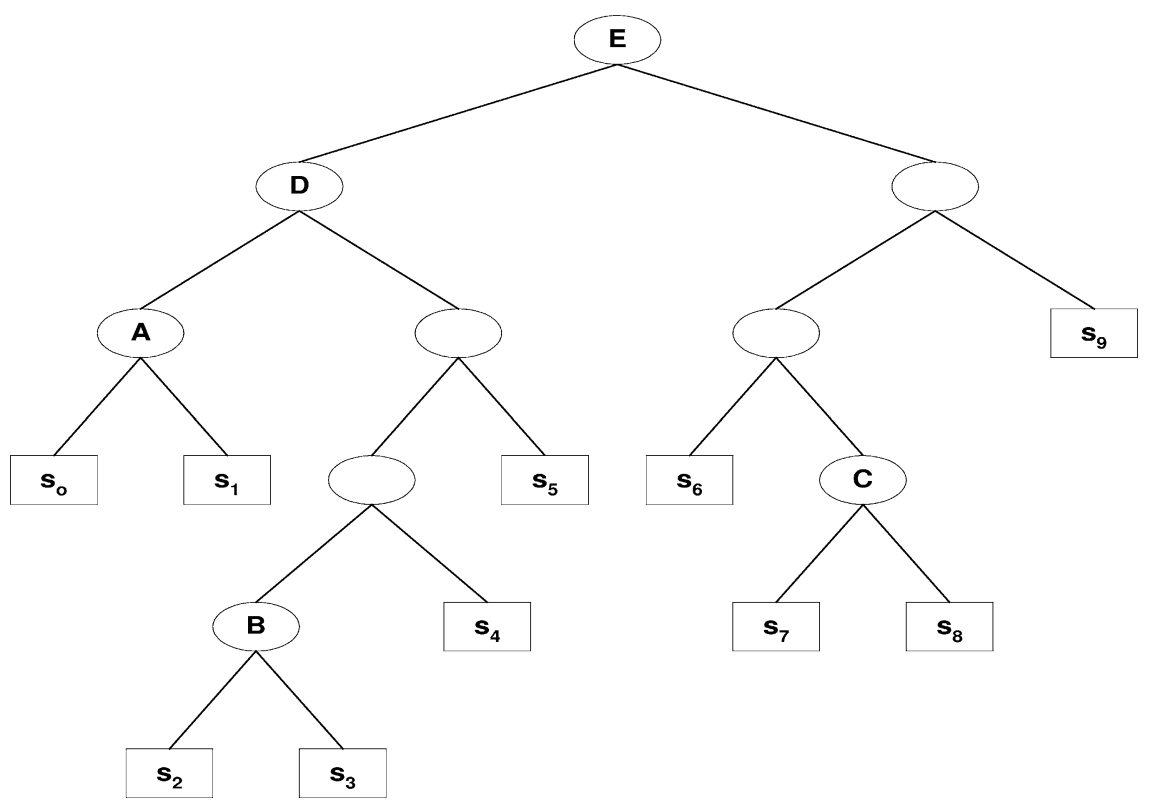

Fig. 1. An example of a Huffman tree. 
Consider the example in Fig. 1. Let the leaf circular nodes of Fig. 1 be denoted by A, B, and C respectively in order. Then the corresponding Huffman codes are 00, 0100 and 101. However, each of these codewords can be represented uniquely by integers whose binary representations are these codewords with 1 as prefix. The corresponding integers for $\mathrm{A}, \mathrm{B}$ and $\mathrm{C}$ are respectively 4 for 100, 20 for 10100 and 13 for 1101 . Along with $s_{0}, s_{1}, \ldots, s_{9}$ if 4,20 and 13 are sent as codes for the circular leaf nodes A, B and C, then the receiver can obtain Huffman codes 00, 0100 and 101 by deleting the attached prefix. These bit patterns will enable the receiver to construct all paths to the circular leaf nodes, and thus reconstruct the Huffman tree. So to reconstruct Huffman tree $T$, we have the following theorem.

Theorem. Let $S$ be the array of symbols in order of appearance in the Huffman tree $T$ and $D$ be the set of integer codes, corresponding to circular leaf nodes, sent to the receiver. Then the receiver can uniquely reconstruct the Huffman tree $T$.

Proof. Let $d_{i}$ be the integer corresponding to a circular leaf node $i$. By deleting the appropriate prefix from the binary representation of $d_{i}$, one can reconstruct the whole path leading to that node uniquely since bit 0 will take to the left and 1 to the right, and there is no ambiguity. Since each internal node of the Huffman tree will appear on the paths of some of these circular leaf nodes, each internal node of the Huffman tree will appear in at least one of these paths. Now external nodes will appear either as left or right sons of the circular leaf nodes, or as one of the sons of circular nodes on the path to the circular leaf nodes. This will result in the construction of a unique Huffman tree $T$ since paths constructed for each $d_{i}$ are unique. Furthermore, the set of symbols $S$ will label the external nodes in an orderly fashion with appropriate symbols.

Once the tree can be constructed all the values of Table 1 can be calculated, and decoding can be successfully accomplished by using a binary search algorithm in the array of cumulative weights.

This gives us the possibility of reducing the overhead further since the number of circular leaf nodes cannot be more than half of the number of symbols. The number $m$ of circular leaf nodes of any truncated
Table 1

The values of $w_{i}$ and $f_{i}$

\begin{tabular}{rrrrrrrrrrr}
\hline$i$ & 0 & 1 & 2 & 3 & 4 & 5 & 6 & 7 & 8 & 9 \\
$w_{i}$ & 4 & 4 & 1 & 1 & 2 & 4 & 4 & 2 & 2 & 8 \\
$f_{i}$ & 4 & 8 & 9 & 10 & 12 & 16 & 20 & 22 & 24 & 32 \\
\hline
\end{tabular}

Huffman tree is 1 more than the number of nodes with 2 internal son nodes. Huffman codes are much more efficient than block codes when corresponding Huffman trees are sparse. In a sparse truncated Huffman tree the number of nodes with 2 internal son nodes can be much less than the total number of internal nodes, which in turn is 1 less than the number of external nodes corresponding to symbols. In the truncated Huffman tree, corresponding to Fig. 1, there are two nodes with 2 internal son nodes, namely nodes labeled $\mathrm{D}$ and $\mathrm{E}$. So in representing the tree we need $\mathrm{O}(n \log n)$ bits for the symbols and $\mathrm{O}(h)$ bits for each circular leaf node, number of which is $\left(\leqslant \frac{1}{2} n\right)$ bounded above by $\mathrm{O}(n)$.

\section{A fast decoding technique}

Chen et al. [1] give weight to a leaf $v$ equal to the number of leaves in a complete binary tree under the node $v$. Every leaf node is assigned a number equal to cumulative weights of all the leaves appearing before it and including itself. So this gives us an opportunity to apply binary search on these cumulative weights to obtain the symbol, for which the corresponding bit pattern has been extracted from the bit stream. At any stage our algorithm takes $h$ bits from the stream. Since a prefix of this string must contain a codeword we will always be able to decode that codeword using the following algorithm. In the algorithm we assume that input bit stream does not contain any error.

\section{Algorithm Decode}

Input: The values $f_{i}, i=0, \ldots, n-1$, of a Huffman tree $T$ with height $h$ and a bit stream $b_{j}, j=1, \ldots$, $N$.

Output: The text symbols $c_{k}, k=1, \ldots, M$, corresponding to the input bit stream.

$/ / j$ is the pointer to the last bit already decoded, $k$ is the pointer to the last decoded symbol, index is the location where the search for $d+1$ fails. Otherwise, 
it is the location at which $d+1$ has been found. $q_{i}$ is a stream of $i 1$ bits. '\#' is the concatenation operator. //

$$
\begin{aligned}
& j \leftarrow 0, k \leftarrow 0 \\
& f_{-1} \leftarrow 0 \\
& \text { while } j<N \text { do } \\
& \text { if }(h<N-j) \text { then } \\
& \quad d \leftarrow b[j+1: j+h] \\
& \text { else } \\
& \quad d \leftarrow b[j+1: N] \# q_{h-N+j} \\
& \text { endif } \\
& \quad \text { binsearch }(f, d+1, \text { index }) \\
& \quad k \leftarrow k+1 \\
& c_{k} \leftarrow f_{\text {index }} \\
& j \leftarrow j+h-\log _{2}\left(f_{\text {index }}-f_{\text {index }-1}\right) \\
& \text { enddo }
\end{aligned}
$$

\section{End of Algorithm Decode}

In decoding the very last symbol of the text there may not be $h$ bits available in the stream in which case $d$ is obtained by appending the bit stream with a sufficient number of 1 bits. It is obvious that in decoding a bit pattern we need to make a binary search among $n$ symbols that requires $\mathrm{O}(\log n)$ comparisons. If there are $m$ codewords, our algorithm will run in $\mathrm{O}(m \log n)$ time.

It may be mentioned here that in order to improve repeated application of Huffman coding it is important to have the overhead of representing a Huffman tree as efficiently as possible. So such efficient repre- sentation of a Huffman header as proposed in this paper gives rise to the opportunity of applying repeated Huffman coding in cases where further compression is desirable.

\section{Acknowledgement}

The authors express their sincere thanks to anonymous referees for pointing out errors in the manuscript and for suggesting improvement of its presentation.

\section{References}

[1] H.-C. Chen, Y.-L. Wang, Y.-F. Lan, A memory-efficient and fast Huffman decoding algorithm, Inform. Process. Lett. 69 (1999) 119-122.

[2] K.L. Chung, Efficient Huffman decoding, Inform. Process. Lett. 61 (1997) 97-99.

[3] R. Hashemian, Memory efficient and high-speed search Huffman coding, IEEE Trans. Comm. 43 (10) (1995) 2576-2581.

[4] D.A. Huffman, A method for construction of minimum redundancy codes, Proc. IRE 40 (1952) 1098-1101.

[5] G.O.H. Katona, T.O.H. Nemetz, Huffman codes and selfinformation, IEEE Trans. Inform. Theory 22 (3) (1978) 337340.

[6] J.N. Mandal, An approach towards development of efficient data compression algorithms and correction techniques, Ph.D. Thesis, Jadavpur University, India, 2000.

[7] R. Schack, The length of a typical Huffman codeword, IEEE Trans. Inform. Theory 40 (4) (1994) 1246-1247.

[8] J.S. Vitter, Design and analysis of dynamic Huffman codes, J. ACM 34 (4) (1987) 825-845. 